\title{
Non-contrast cardiac computed tomography can accurately detect chronic myocardial infarction: Validation study
}

\author{
Mohit Gupta, MD, a Jigar Kadakia, MD, , Yalcin Hacioglu, MD, ${ }^{\mathrm{a}}$ \\ Naser Ahmadi, MD, ${ }^{\text {a }}$ Amish Patel, MD, ${ }^{a}$ Taeyoung Choi, MD, ${ }^{a}$ \\ Gregg Yamada, $M D{ }^{b}$ and Matthew Budoff, $M^{a}$
}

Background. This study evaluates whether non-contrast cardiac computed tomography (CCT) can detect chronic myocardial infarction (MI) in patients with irreversible perfusion defects on nuclear myocardial perfusion imaging (MPI).

Methods. One hundred twenty-two symptomatic patients with irreversible perfusion defect $(N=62)$ or normal MPI $(N=60)$ underwent coronary artery calcium (CAC) scanning. MI on these non-contrast CCTs was visually detected based on the hypo-attenuation areas (dark) in the myocardium and corresponding Hounsfield units (HU) were measured.

Results. Non-contrast CCT accurately detected MI in 57 patients with irreversible perfusion defect on MPI, yielding a sensitivity of $92 \%$, specificity of $72 \%$, negative predictive value (NPV) of $90 \%$, and a positive predictive value (PPV) of $77 \%$. On a per myocardial region analysis, non-contrast CT showed a sensitivity of $70 \%$, specificity of $85 \%$, NPV of $91 \%$, and a PPV of 57\%. The ROC curve showed that the optimal cutoff value of $L V$ myocardium HU to predict MI on non-contrast CCT was 21.7 with a sensitivity of $97.4 \%$ and specificity of $99.7 \%$.

Conclusion. Non-contrast CCT has an excellent agreement with MPI in detecting chronic MI. This study highlights a novel clinical utility of non-contrast CCT in addition to assessment of overall burden of atherosclerosis measured by CAC. (J Nucl Cardiol 2011;18:96-103.)

Key Words: Myocardial perfusion imaging: SPECT $\cdot$ infarction $\cdot$ myocardial $\cdot$ computed tomography $(\mathrm{CT}) \cdot$ coronary artery disease $\cdot$ diagnostic $\cdot$ prognostic application

\section{See related editorial, pp. $21-23$}

\section{INTRODUCTION}

The principal cause of morbidity and mortality in all industrialized nations is coronary artery disease (CAD).

From the Division of Cardiology, ${ }^{\mathrm{a}}$ Los Angeles Biomedical Research Institute at Harbor UCLA Medical Center, Torrance, CA. Division of Cardiology, ${ }^{\text {b }}$ Stanford University School of Medicine, Stanford, CA.

Received for publication Apr 15, 2010; final revision accepted Oct 25, 2010.

Reprint requests: Mohit Gupta, MD, Division of Cardiology, Los Angeles Biomedical Research Institute at Harbor UCLA Medical Center, 1124 W Carson St., Torrance, CA 90502; mohit_gupta13@ yahoo.com.

$1071-3581 / \$ 34.00$

Copyright (C 2010 The Author(s). This article is published with open access at Springerlink.com

doi:10.1007/s12350-010-9314-3
In approximately half of these patients, the initial presentation of CAD is either myocardial infarction (MI) or death. ${ }^{1}$ Unrecognized MI leads to less effective management and increased major adverse cardiac events (MACE). Population-based studies have revealed that one-fourth of MIs demonstrated by Q waves on the ECG are clinically unrecognized and estimates of the frequency of unrecognized MI detected in the Framingham study may well underestimate the true prevalence of the condition. ${ }^{2}$ The 10 -year mortality from an unrecognized MI has been estimated to be $45 \%$ to $55 \%^{3,4}$ comparable to or even higher than in patients with diagnosed MI. Given the implications of unrecognized MI, studies are needed to address screening strategies, risk stratification, and the role of post-infarction therapies in these patients. $^{5}$

Nuclear myocardial perfusion imaging (MPI) has been widely used as a non-invasive method for the diagnosis of CAD. In comparative studies with autopsy findings, thallium (Tl)-201 myocardial single-photon emission computed tomography (SPECT) has been 
shown to accurately assess the extent of $\mathrm{MI}^{6}$ and myocardial viability for the selection of therapy and for predicting prognosis. ${ }^{7-9}$ Although rest Tc-99m-methoxyisobutyl isonitrile (MIBI) underestimates the extent of viable myocardium compared with Tl-201 imaging, however several studies show that sublingual administration of nitroglycerin before a rest Tc-99m-MIBI study improves the detection of defect reversibility in exercise-induced perfusion defects and thus improves the assessment of myocardial viability in comparison with the standard Tc-99m-MIBI exercise-rest protocol. ${ }^{10,11}$

Coronary artery calcium (CAC) score measured through non-contrast cardiac computed tomography (CCT) scanning has been shown to predict future coronary events in both asymptomatic and symptomatic patients. ${ }^{12}$ Non-contrast CCT has also been used for assessing the pericardial fat, ${ }^{13}$ liver fat, and determining the field of view for CCT angiography (CCTA) to reduce radiation dose. ${ }^{14}$ The potential role of non-enhanced CCT to detect chronic MI, however, has not been well studied. Areas of chronic left ventricular (LV) infarction are readily apparent on non-contrast CCT as regions of hypo-attenuation either due to deposition of fibrous or adipose tissue $^{15,16}$ or probably reduced capillary density. ${ }^{17}$

We conducted this study to evaluate the accuracy and feasibility of using non-contrast CCT to detect chronic MI in patients with irreversible perfusion defect on MPI. We also define the optimal cutoff value for LV myocardium Hounsfield unit (HU) to detect chronic MI.

\section{METHODS}

\section{Study Population}

Our study included 122 consecutive symptomatic patients (with exertional angina or dyspnea) who were referred for

Table 1. Demographics of the total study population $(\mathrm{N}=122)$

\begin{tabular}{ll}
\hline Age (years) & $63.5 \pm 11.9$ \\
Sex (M/F) & $71(58.2 \%) / 51(41.8 \%)$ \\
Risk factors & \\
$\quad$ Hypertension & $83(68.0 \%)$ \\
Cholesterol & $81(66.4 \%)$ \\
Diabetes & $35(28.7 \%)$ \\
Smoker & $17(13.9 \%)$ \\
Family history & $58(47.5 \%)$ \\
Race & \\
Caucasian & $66(54.0 \%)$ \\
Hispanic & $26(21.3 \%)$ \\
Asian & $24(19.7 \%)$ \\
African American & $6(4.9 \%)$ \\
\hline
\end{tabular}

coronary CAC scanning at our institution after undergoing an MPI study. Patients with reversible perfusion defects on MPI were excluded. Subjects with arrhythmia, renal failure, or hemodynamic instability were excluded. The characteristics of the study population are summarized in Tables 1 and 2 .

Demographic data and clinical history were retrieved from the clinical records for all patients in the study group. Information about any evidence of previous clinical MI was also collected. MI in these patients was diagnosed by electrocardiogram findings (ST-segment elevation or depression, pathologic Q waves, new onset of left bundle branch block) and abnormal levels of cardiac enzymes.

\section{Non-Contrast CCT Acquisition and Analysis}

All CCTs were performed using 64-multidetector CT scanner (GE Healthcare). Following a scout radiograph of the chest (antero-posterior and lateral), the coronary arteries were imaged with $30-40$ contiguous and $2.5-\mathrm{mm}$ slices during middiastole using ECG-triggering during a 10-s breath hold. CAC scoring acquisition was reconstructed at $75 \%$ of the $\mathrm{R}-\mathrm{R}$ interval with the use of axial planes and multiplanar reconstruction. All the non-enhanced CCT images were assessed by two experienced readers for presence and location of MI on Advantage Windows (AW) 4.4 Workstation (General Electric Medical Systems, Milwaukee, WI, USA).

Longitudinal views of axial images were evaluated from base to apex to assess the septal, antero-apical, and lateral LV wall; coronal images were used to assess the inferior wall. MI on non-enhanced CCT was visually detected based on the areas of hypo-attenuation (dark) in the myocardium. Hypo-attenuation was assessed by the consensus of two experienced observers who blinded to the results of the MPI. The mean HU of normal and hypo-attenuated area were measured in the septal, antero-apical, lateral, and inferior LV walls. A standard region of interest (ROI) of $1 \mathrm{~cm}^{2}$ was used for the normal myocardium; and for areas with small hypo-attenuation, smaller ROI was used (Figure 1).

\section{MPI Acquisition and Analysis}

All the MPI scans were performed by the referring physicians at their respective offices. A standard imaging protocol as endorsed by ASNC was used for all patients. ${ }^{18}$ All patients underwent rest and stress imaging with technetium-99m-sestamibi (MIBI). Patients who could exercise underwent treadmill stress using the Bruce protocol to reach $85 \%$ of maximum predicted heart rate. Patients who could not exercise underwent pharmacological stress using dipyridamole at $142 \mathrm{mg} / \mathrm{kg} / \mathrm{min}$ infused over $4 \mathrm{~min}$. Patients were asked to terminate beta-blocker $24 \mathrm{~h}$ prior to testing when desirable. For resting studies, patients were injected with $9-10 \mathrm{mCi}$ of technetium-99m-sestamibi and SPECT images were acquired 30-45 min after resting injection. All SPECT studies were acquired on a commercially available camera and computer using a 180-degree arc, high-resolution parallel-hole collimator; and 64 stops with $20 \mathrm{~s}$ per stop for a total imaging time of 25 min. All images were acquired using a $64 \times 64$ matrix. 
Table 2. Characteristics of the groups with normal and irreversible perfusion defect on MPI

\begin{tabular}{lccc}
\hline \multicolumn{1}{c}{ Variable } & $\begin{array}{c}\text { Normal myocardial } \\
\text { perfusion (N= 60) }\end{array}$ & $\begin{array}{c}\text { Irreversible perfusion } \\
\text { defect (N= 62) }\end{array}$ & P-value \\
\hline Age (years) & $62 \pm 11$ & $64 \pm 12$ & .2 \\
Gender (male) & 30 & 42 & .046 \\
Hypertension & $67 \%$ & $69 \%$ & .7 \\
Hypercholesterolemia & $65 \%$ & $68 \%$ & .7 \\
Diabetes mellitus & $27 \%$ & $31 \%$ & .6 \\
Family history of CHD & $62 \%$ & $34 \%$ & .002 \\
Smoking status & $8 \%$ & $19 \%$ & .08 \\
Known CAD & $35 \%$ & $50 \%$ & .09 \\
Septal LV wall HU & $37 \pm 19$ & $14 \pm 19$ & .0001 \\
Antero-apical LV wall HU & $42 \pm 16$ & $16 \pm 26$ & .0001 \\
Lateral wall HU & $38 \pm 19$ & $13 \pm 28$ & .0001 \\
Inferior wall HU & $36 \pm 26$ & $8 \pm 27$ & .0001 \\
\hline
\end{tabular}

$H U$, Hounsfield unit.
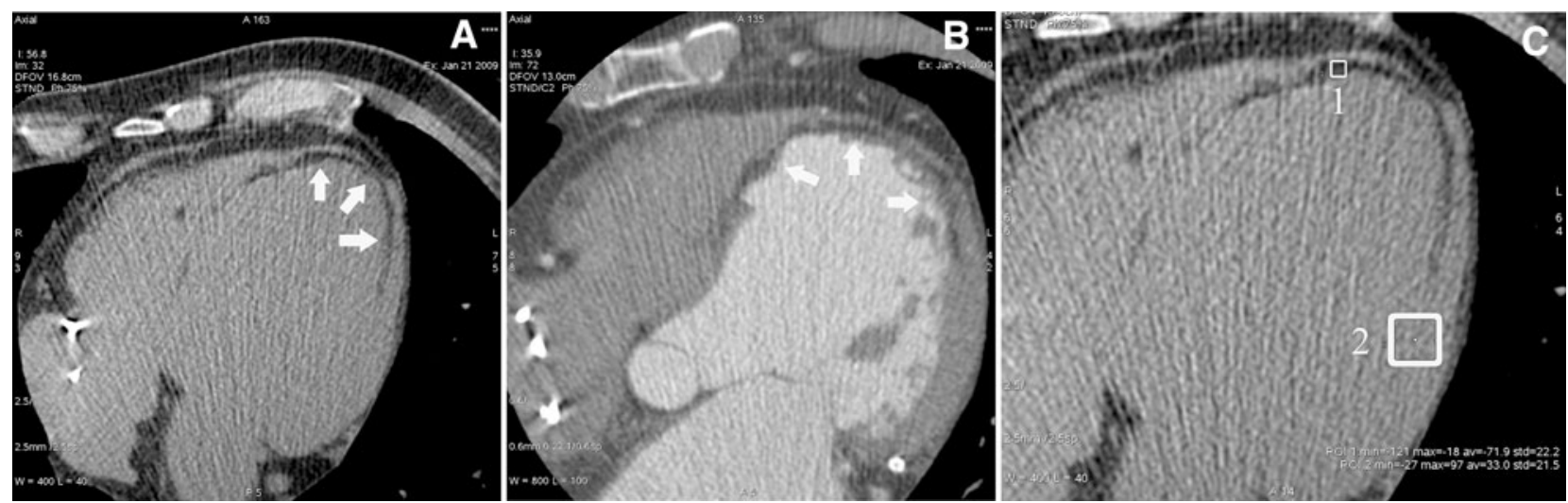

Figure 1. (A) Axial, non-contrast CCT image demonstrating thin curvilinear hypo-attenuation (arrows) within distal septal, apical, and distal lateral LV myocardium in subendocardial distribution. (B) Axial, contrast-enhanced CCT image of the same patient shows thinning of associated portions (arrows) of the LV myocardium. (C) Sample placement of ROI within infarcted and normal myocardium on non-contrast CCT image to assess mean HU. ROI 1 mean HU $=-71.9$ (infarct), ROI 2 mean $\mathrm{HU}=33$ (normal myocardium).

Patients underwent symptom-limited exercise Bruce protocol. At peak heart rate, $25-30 \mathrm{mCi}$ of technetium-99m-sestamibi (dose was weight based) was injected. Patient then underwent post-stress SPECT image acquisition.

The post-stress and rest MIBI scans were interpreted using visual assessment of perfusion abnormalities by the referring physicians. All the readers were nuclear board certified and blinded to the results of CT. The short axis data were displayed in polar map format, with the maps divided into 17 segments. The 17 segments were allocated to the territories of the different coronary arteries as previously described. ${ }^{19}$ For the sake of comparison with $\mathrm{CT}$, the nuclear study results were then extrapolated into a 4-region model with the LV wall divided into septal, antero-apical, lateral, and inferior as done on CCT. Gating was not performed in all the MPI studies and thus regional wall motion was not assessed.

\section{Statistical Analysis}

Categorical variables are presented as frequencies and percentages, and continuous variables as mean \pm standard deviations (SD). MPI served as the reference standard for the diagnosis of regional MI. The area under the receiver operator characteristics (ROC) curve was calculated for MI assessed by non-contrast CCT to identify irreversible perfusion defect detected by MPI. Diagnostic performance and predictive value of non-contrast CCT for the diagnosis of regions showing MI 
compared with the reference standard was evaluated on a per patient level and per region level and expressed as sensitivity, specificity, positive predictive value (PPV), and negative predictive value (NPV) and their corresponding 95\% confidence intervals.

The mean HU of normal and hypo-attenuated area in the septal, antero-apical, lateral, and inferior LV walls were compared to their corresponding areas in MPI and a second ROC was used to determine the myocardial HU threshold to detect MI using non-enhanced CCT. Intra- and inter-observer variability was calculated using intra-class correlation coefficient (ICC) in a two-way mixed effects model, and Bland \& Altman models. All statistical analyses were performed using PASW 18.0 (SPSS Institute Inc., Chicago, IL, USA).

\section{RESULTS}

A total of 122 patients were studied, including 62 patients $(51 \%)$ with an irreversible perfusion defect on MPI and 60 patients with a normal MPI. The mean age for this patient cohort was $63.5 \pm 12$ years and 71(58\%) were males.

Out of the 62 patients with irreversible perfusion defect on MPI, non-contrast CCT accurately detected infarct in 57 subjects. Thus, on a per patient level and using MPI as the reference standard, non-contrast CCT showed a sensitivity of $92 \%$ (95\% CI, 82.2-97.3), specificity of $72 \%$ (95\% CI, 59.2-82.9), NPV of $90 \%$ (95\% CI, 77.8-96.6), and a PPV of 77\% (95\% CI, 65.485.8 ), to detect regions of MI. On a per region level, non-contrast CCT showed a sensitivity of $70 \%(95 \% \mathrm{CI}$, 59.8-78.1), specificity of $85 \%$ (95\% CI, 81.4-88.8), NPV of $91 \%(95 \% \mathrm{CI}, 87.6-93.8)$, and a PPV of $57 \%(95 \%$ CI, 47.6-65.3) to detect regions of MI on MPI (Table 3). There was good agreement in the identification of hypoenhanced regions between MPI and non-contrast CCT (Figures 2 and 3).

The mean HU of the regions correctly identified as infarct or normal was calculated to be $5.1 \pm 18.3$ and $46.2 \pm 8.2(P<.0001)$, respectively. Based on a ROC curve, a reference HU of 21.7 can be used to detect infarcts on non-contrast CCT with a sensitivity of $97.4 \%$ and specificity of $98.7 \%$ (Figure 4).

In a sub-study analysis, intra- and inter-observer variability of 25 patients with and without irreversible perfusion defect detected by MPI was assessed using visual and corresponding HU criteria methods on CCT. The intra- and inter-observer variability of CCT measured normal and hypo-attenuation segments were low (coefficient of variability of $2 \%$ for intra-observer and $6 \%$ for inter-observer variability). Furthermore, there was good intra- and inter-observer agreement to assess normal vs hypo-attenuated segments in CT (ICC: 0.78 and 0.811 , respectively, $P=.0001$ ) (Table 4 ).

In a subgroup of 38 patients, with clinical evidence of prior MI diagnosed on the basis of electrocardiogram findings or abnormal levels of cardiac enzymes (as described in "Methods" section), hypo-attenuation on CAC scan was present in 30 patients yielding a sensitivity of $79 \%$ to detect chronic MI. Out of these 38 patients with clinical MI, irreversible perfusion defects on MPI were present in 32 patients.

\section{DISCUSSION}

Contrast-enhanced CCTA has been shown to be comparable to MPI and contrast-enhanced cardiac magnetic resonance (CMR) in detecting fixed perfusion defect or scar as areas of late contrast enhancement. ${ }^{20-23}$ Hypo-enhanced regions on contrast-enhanced CCTA have been shown to closely relate to delayed enhancement of iodinated contrast. ${ }^{24,25}$ The aim of this study was to evaluate the ability and feasibility of non-contrast CCT scans to detect chronic MI in patients with irreversible perfusion defect on MPI.

Chronic MI is characterized by a process of ventricular remodeling, fatty replacement (rarefaction), and in rare cases calcification. Small studies have documented that the presence of fat and/or calcification in the LV-free wall on non-contrast CCT is consistent with MI. ${ }^{16,26,27}$ Subsequently, areas of infarction are readily apparent on non-contrast CCT as regions of hypoattenuation either due to reduced capillary density ${ }^{17}$ or deposition of adipose tissue. ${ }^{15,28} \mathrm{CT}$ imaging features of MI include thin linear or curvilinear fat attenuation within LV myocardium, most commonly sub-endocardial, often associated with $\mathrm{LV}$ wall thinning, and/or calcification. ${ }^{16}$ Furthermore, the presence of LV myocardial fat deposition in sites of myocardial scarring has

Table 3. Accuracy of non-contrast CCT to detect chronic MI as compared to irreversible perfusion defects on MPI as the reference

$\begin{array}{cccc}\begin{array}{c}\text { Sensitivity } \\ (95 \% \text { CI })\end{array} & \begin{array}{c}\text { Specificity } \\ (95 \% \text { CI })\end{array} & \text { PPV } & \text { NPV } \\ (95 \% \text { CI }) & (95 \% \text { CI) }\end{array}$

\begin{tabular}{lllll} 
Per patient analysis & $91.9 \%(82.2-97.3)$ & $72.1 \%(59.2-82.9)$ & $76.7 \%(65.4-85.8)$ & $89.8 \%(77.8-96.6)$ \\
Per region analysis & $69.5 \%(59.8-78.1)$ & $85.4 \%(81.4-88.8)$ & $56.6 \%(47.6-65.3)$ & $91.1 \%(87.6-93.8)$ \\
\hline
\end{tabular}



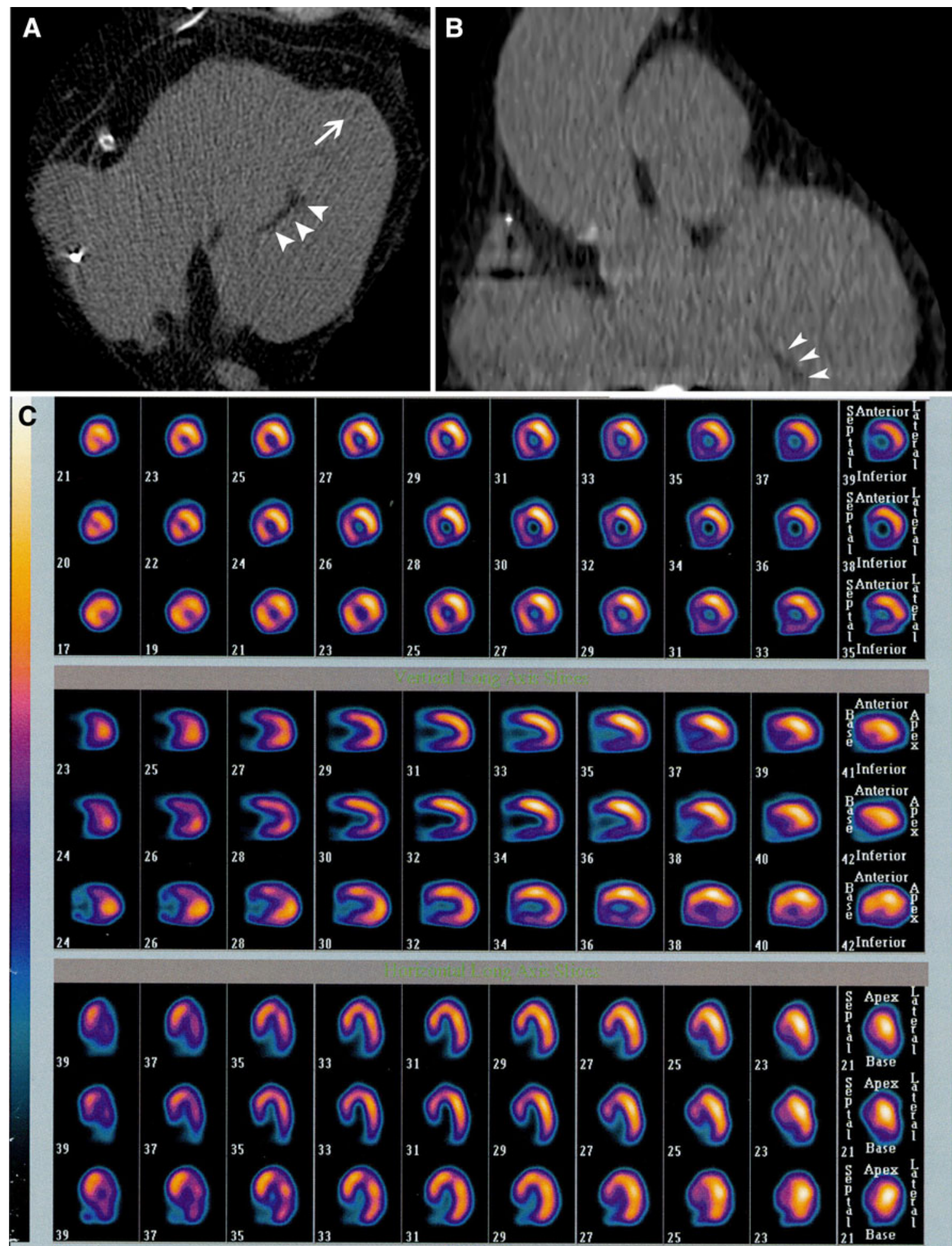

Top Rows

Stress Summed Recon S.A.

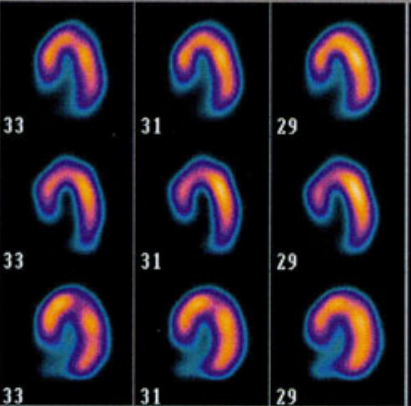

Middle Rows

Rest Summed Recon S.A.

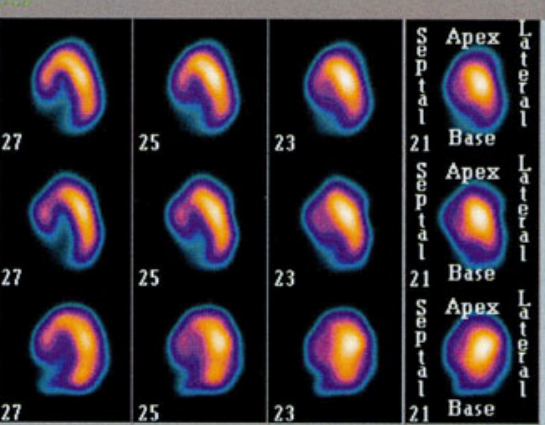

Bottom Rows Stress PRONE Short Axis

Figure 2. (A) Axial, non-contrast CCT image demonstrating an infero-septal (arrowheads) and anterior (arrow) wall infarct. (B) Coronal, non-contrast CCT image confirming the infero-septal (arrowheads) infarct. Lead artifact makes the assessment of infarct difficult in this view. (C) Nuclear MPI of the same patient demonstrating the fixed perfusion defects. 


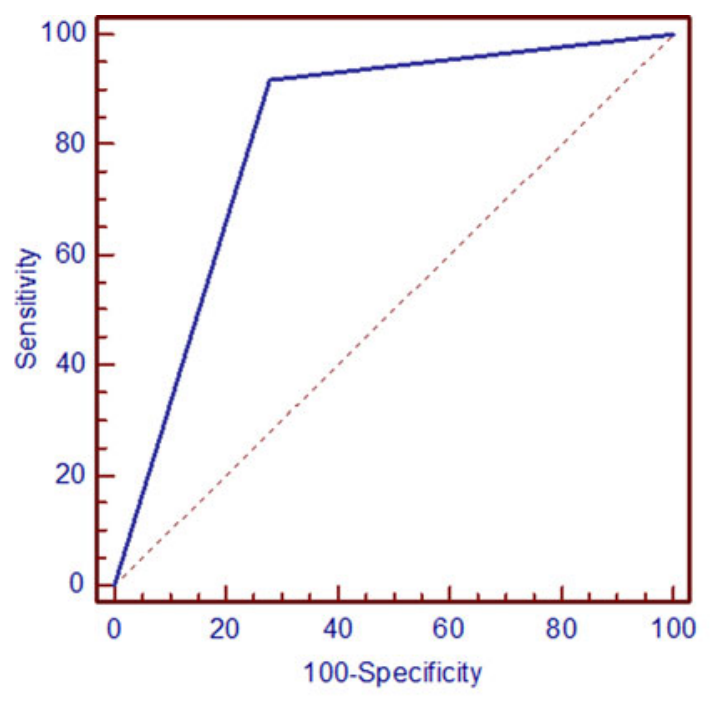

\begin{tabular}{|c|c|c|c|}
\hline Model & AUC (SE) & 95\%CI & P \\
\hline CT diagnosed perfusion defect & $0.82(0.03)$ & $0.74-0.88$ & 0.0001 \\
\hline
\end{tabular}

Figure 3. Area under ROC curve for accuracy of non-contrast CCT to detect MI diagnosed by MPI.

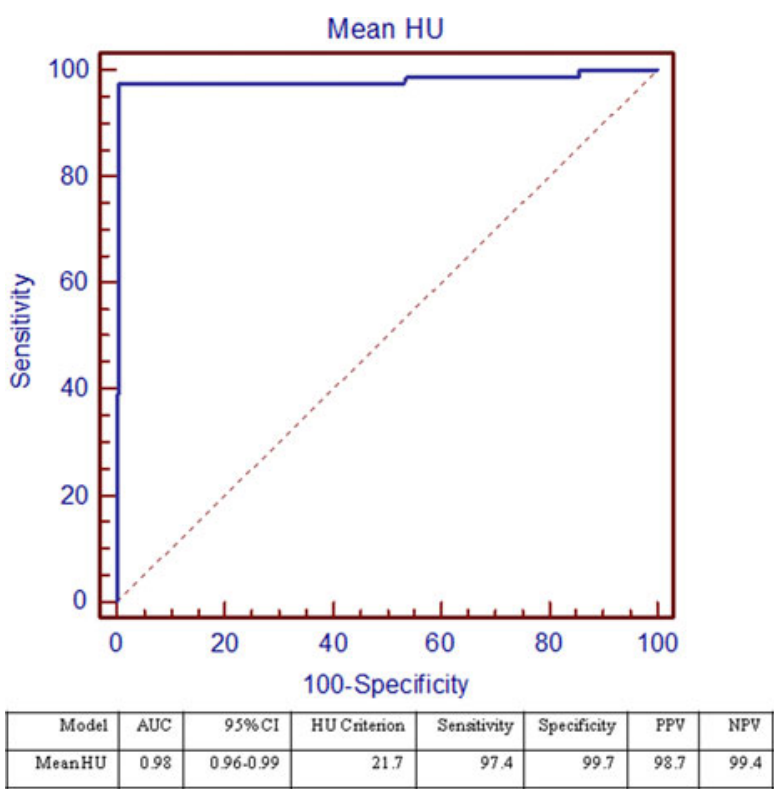

Figure 4. ROC curve for accuracy of HU criterion to detect MI on non-contrast CCT.

been reported to be associated with severe heart failure. $^{27}$

The preliminary results of our study demonstrate that the non-contrast CCT performed for standard CAC scoring has an excellent agreement with MPI in detecting irreversible perfusion defects. Despite a moderate specificity of $72 \%$, CAC showed an excellent sensitivity of $92 \%$ to detect irreversible perfusion
Table 4. Intra- and inter-observer variability to detect MI on non-contrast CCT

\begin{tabular}{ccccc}
\hline Variable & $D$ & $95 \%$ CI & \multicolumn{1}{c}{ ICC } & $P$-value \\
\hline Intra-observer & 0.02 & -0.05 to 0.09 & 0.78 & .0001 \\
Inter-observer & 0.06 & -0.01 to 0.13 & 0.81 & .0001
\end{tabular}

$D$, mean ratio difference; ICC, intra-class correlation coefficient.

defects on. The attenuation levels in the infarcted myocardium were lower than the normal myocardium $(5.1 \pm 18.3$ and $46.2 \pm 8.2$, respectively $)$ and the difference was statistically significant $(P<.0001)$. We also determined the optimal cutoff value for LV myocardium HU to detect chronic MI as 21.7, yielding a sensitivity of $97.4 \%$ and specificity of $99.7 \%$ based on the ROC curve. This cutoff can be used as an objective method of diagnosing MI on CCT in daily clinical practice with high accuracy (AUC-0.98, 95\% CI, 0.960.99).

CAC score measured through non-contrast CCT scanning has been well validated to predict future coronary events in both asymptomatic and symptomatic patients. ${ }^{12}$ This study highlights a novel clinical utility of non-contrast CCT in addition to assessment of overall burden of atherosclerosis measured by CAC, and provides evidence that it can accurately detect chronic MI. We illustrate the importance of complete review of the left ventricle apart from calcium scoring as an addition to the role of non-contrast $\mathrm{CT}$. This application can further be used to detect occult scars in asymptomatic population undergoing CAC scan for risk stratification.

Silent or unrecognized MI, which was once thought to be an unusual presentation of CAD, is now widely recognized as a prominent manifestation of the disease process. Past cohort studies have demonstrated that $25 \%-40 \%$ of MIs are clinically unrecognized. ${ }^{29}$ These asymptomatic infarctions escape detection until ultimately an ECG is performed for screening or other clinical purposes. The true prevalence of unrecognized MI may be even higher, owing to the insensitivity of $Q$ waves. $^{5}$ Despite its benign clinical presentation the prognosis for unrecognized MI is about as serious as that for recovered symptomatic infarction for all cause and cardiovascular mortality. ${ }^{2}$ Thus, there is a need for a cost effective screening mechanism for early diagnosis of prior MI to promote early management and potentially improved outcomes.

Although other imaging techniques like late gadolinium enhancement (LGE) on contrast-enhanced CMR can detect and characterize MI that is missed by clinical history, ECG, conventional wall motion, ${ }^{30,31}$ or nuclear 
scintigraphic techniques, ${ }^{32}$ non-contrast CCT is a less expensive, non-invasive method for detecting unrecognized MI. There is no incremental radiation or scanning required beyond the CAC scan, so one can simultaneously evaluate for both prior MI and CAC with one scan, potentially adding incremental value to the CAC scan at no cost.

The presence of LGE on CMR has been shown to provide incremental prognostic value to MACE and cardiac mortality beyond common clinical, angiographic, and functional predictors. ${ }^{33,34}$ These occult myocardial scars have been associated with a high risk of future cardiac events, and therefore identify a subpopulation of patients who may benefit from more intensive medical or revascularization treatment strategies. Similarly, the presence and extent of MI as detected by a non-contrast CCT could also be a predictor of MACE and cardiac death. The presence of hypoattenuation may provide incremental prognostic value beyond traditional risk factors and markers of subclinical atherosclerosis. Non-contrast CCT may also represent a better standard for unrecognized MI than ECG for future population-based studies. Whether these findings can result in better patient outcomes by guiding management decisions, such as implantation of ICDs, coronary revascularization, or intensive medical treatment, requires additional study.

\section{LIMITATION}

The primary limitation of this study is that we used irreversible perfusion defects on standard rest MIBI as the reference for detecting chronic MI on CAC scan. However, it should be noted that rest Tc-99m-MIBI underestimates the extent of viable myocardium compared with Tl-201 imaging, and thus some of the irreversible perfusion defects might represent hibernating viable myocardium. Thus, in this study some of the true positives on CAC scan could actually have been false positives. Sublingual administration of NTG before a rest Tc-99m-MIBI study would be a better reference standard with improved assessment of myocardial viability.

Although MPI has been validated as an accurate tool to identify the presence of MI, it is known to give a large number of false-positive results, primarily because of attenuation artifacts. Also, smaller infarcts might be underestimated or missed by MPI due to its limited resolution. Thus, some non-contrast CCT scans may have been classified as false negatives or false positives due to false nuclear results. CMR imaging has been proven to be more sensitive than MPI in the detection of small and occult MIs. Therefore, additional studies using
CMR with delayed enhancement as the reference standard are warranted.

\section{CONCLUSION}

This study demonstrates that the non-contrast CCT has an excellent agreement with MPI in detecting chronic MI. Further prospective studies are warranted to evaluate the role of non-contrast CCT in infarct assessment for the management of at-risk patients.

\section{Acknowledgment}

None of the authors have received any funding for this study from any institution.

\section{Open Access}

This article is distributed under the terms of the Creative Commons Attribution Noncommercial License which permits any noncommercial use, distribution, and reproduction in any medium, provided the original author(s) and source are credited.

\section{References}

1. Kannel WB, Schatzkin A. Sudden death: Lessons from subsets in population studies. J Am Coll Cardiol 1985;5:141B-9B.

2. Kannel WB, Cupples LA, Gagnon DR. Incidence, precursors and prognosis of unrecognized myocardial infarction. Adv Cardiol 1990;37:202-14.

3. Yano K, MacLean CJ. The incidence and prognosis of unrecognized myocardial infarction in the Honolulu, Hawaii, Heart Program. Arch Intern Med 1989;149:1528-32.

4. Kannel WB, Abbott RD. Incidence and prognosis of unrecognized myocardial infarction: an update on the Framingham study. N Engl J Med 1984;311:1144-7.

5. Sheifer SE, Manolio TA, Gersh BJ. Unrecognized myocardial infarction. Ann Intern Med 2001;135:801-11.

6. Ide H, Yamada H, Ohkawa S, Sugiura M, Harumi K. Quantification of the extent of myocardial infarction by thallium-201 single photon emission computed tomography: Comparisons with post-mortem findings. J Cardiol 1990;20:1-14.

7. Nakayama M, Ohkawa S, Tanno M, Yamada H, Mashima S. Myocardial viability in cases with persistent perfusion defects on the dipyridamole thallium-201 scintigram. A comparative study with autopsy findings. Jpn Heart J 1996;37:301-16.

8. Bonow RO, Disizian V. Thallium 201 for assessment of myocardial viability. Semin Nucl Med 1991;21:230-41.

9. Brown KA. Prognostic value of thallium-201 myocardial perfusion imaging. A diagnostic tool comes of age. Circulation 1991;83:363-81.

10. Batista JF, Pereztol O, Valdés JA, Sánchez E, Stusser R, Rochela LM, et al. Improved detection of myocardial perfusion reversibility by rest-nitroglycerin Tc-99m-MIBI: Comparison with TI201 reinjection. J Nucl Cardiol 1999;6:480-6.

11. Maurea S, Cuocolo A, Soricelli A, Castelli L, Nappi A, Squame F, et al. Enhanced detection of viable myocardium by technetium- 
99m-MIBI imaging after nitrate administration in chronic coronary artery disease. J Nucl Med 1995;36:1945-52.

12. Budoff MJ, Achenbach S, Blumenthal RS, Carr JJ, Goldin JG, Greenland P, et al. Assessment of coronary artery disease by cardiac computed tomography: A scientific statement from the American Heart Association Committee on Cardiovascular Imaging and Intervention, Council on Cardiovascular Radiology and Intervention, and Committee on Cardiac Imaging Council on Clinical Cardiology. Circulation 2006;114:1761-91.

13. Dey D, Suzuki Y, Suzuki S, Ohba M, Slomka PJ, Polk D, et al. Automated quantitation of pericardiac fat from noncontrast CT. Invest Radiol 2008;43:145-53.

14. Gopal A, Budoff MJ. A new method to reduce radiation exposure during multi-row detector cardiac computed tomographic angiography. Int J Cardiol 2009;132:435-6.

15. Goldfarb JW, Arnold S, Roth M, Han J. T1-weighted magnetic resonance imaging shows fatty deposition after myocardial infarction. Magn Reson Med 2007;57:828-34.

16. Zafar HM, Litt HI, Torigian DA. CT imaging features and frequency of left ventricular myocardial fat in patients with CT findings of chronic left ventricular myocardial infarction. Clin Radiol 2008;63:256-62.

17. Rodríguez-Granillo GA, Rosales MA, Renes P, Diez E, Pereyra J, Gomez E, et al. Chronic myocardial infarction detection and characterization during coronary artery calcium scoring acquisitions. J Cardiovasc Comput Tomogr 2010;4:99-107.

18. Hansen CL, Goldstein RA, Berman DS, Churchwell KB, Cooke $\mathrm{CD}$, Corbett JR, et al. Myocardial perfusion and function single photon emission computed tomography. J Nucl Cardiol 2006;13:e97-120.

19. Cerqueira MD, Weissman NJ, Dilsizian V, Jacobs AK, Kaul S, Laskey WK, American Heart Association Writing Group on Myocardial Segmentation and Registration for Cardiac Imaging, et al. Standardized myocardial segmentation and nomenclature for tomographic imaging of the heart: A statement for healthcare professionals from the Cardiac Imaging Committee of the Council on Clinical Cardiology of the American Heart Association. Circulation 2002;105:539-42.

20. Paul JF, Wartski M, Caussin C, Sigal-Cinqualbre A, Lancelin B, Angel $\mathrm{C}$, et al. Late defect on delayed contrast-enhanced multidetector row CT scans in the prediction of SPECT infarct size after reperfused acute myocardial infarction: Initial experience. Radiology 2005;236:485-9.

21. Henneman MM, Schuijf JD, Jukema JW, Lamb HJ, de Roos A, Dibbets $\mathrm{P}$, et al. Comprehensive cardiac assessment with multislice computed tomography: Evaluation of left ventricular function and perfusion in addition to coronary anatomy in patients with previous myocardial infarction. Heart 2006;92:1779-83.

22. Baks T, Cademartiri F, Moelker AD, Weustink AC, van Geuns RJ, Mollet NR, et al. Multislice computed tomography and magnetic resonance imaging for the assessment of reperfused acute myocardial infarction. J Am Coll Cardiol 2006;48:144-52.
23. Gerber BL, Belge B, Legros GJ, Lim P, Poncelet A, Pasquet A, et al. Characterization of acute and chronic myocardial infarcts by multidetector computed tomography: Comparison with contrastenhanced magnetic resonance. Circulation 2006;113:823-33.

24. Mahnken AH, Bruners P, Katoh M, Wildberger JE, Gunther RW, Buecker A. Dynamic multi-section CT imaging in acute myocardial infarction: Preliminary animal experience. Eur Radiol 2006;16:746-52.

25. Lardo AC, Cordeiro MA, Silva C, Amado LC, George RT, Saliaris AP, et al. Contrast-enhanced multidetector computed tomography viability imaging after myocardial infarction: Characterization of myocyte death, microvascular obstruction, and chronic scar. Circulation 2006;113:394-404.

26. Winer-Muram HT, Tann M, Aisen AM, Ford L, Jennings SG, Bretz R. Computed tomography demonstration of lipomatous metaplasia of the left ventricle following myocardial infarction. J Comput Assist Tomogr 2004;28:455-8.

27. Baroldi G, Silver MD, De Maria R, Parodi O, Pellegrini A. Lipomatous metaplasia in left ventricular scar. Can J Cardiol 1997;13:65-71.

28. Su L, Siegel JE, Fishbein MC. Adipose tissue in myocardial infarction. Cardiovasc Pathol 2004;13:98-102.

29. Sheifer SE, Gersh BJ, Yanez ND 3rd, Ades PA, Burke GL, Manolio TA. Prevalence, predisposing factors, and prognosis of clinically unrecognized myocardial infarction in the elderly. J Am Coll Cardiol 2000;35:119-26.

30. Kim RJ, Fieno DS, Parrish TB, Harris K, Chen EL, Simonetti O, et al. Relationship of MRI delayed contrast enhancement to irreversible injury, infarct age, and contractile function. Circulation 1999;100:1992-2002.

31. Ricciardi MJ, Wu E, Davidson CJ, Choi KM, Klocke FJ, Bonow $\mathrm{RO}$, et al. Visualization of discrete microinfarction after percutaneous coronary intervention associated with mild creatine kinaseMB elevation. Circulation 2001;103:2780-3.

32. Wagner A, Mahrholdt H, Holly TA, Elliott MD, Regenfus M, Parker M, et al. Contrast-enhanced MRI and routine single photon emission computed tomography (SPECT) perfusion imaging for detection of subendocardial myocardial infarcts: An imaging study. Lancet 2003;361(9355):374-9.

33. Kwong RY, Chan AK, Brown KA, Chan CW, Reynolds HG, Tsang S, et al. Impact of unrecognized myocardial scar detected by cardiac magnetic resonance imaging on event-free survival in patients presenting with signs or symptoms of coronary artery disease. Circulation 2006;113:2733-43.

34. Kwong RY, Sattar H, Wu H, Vorobiof G, Gandla V, Steel K, et al. Incidence and prognostic implication of unrecognized myocardial scar characterized by cardiac magnetic resonance in diabetic patients without clinical evidence of myocardial infarction. Circulation 2008;118:1011-20. 